\title{
Lessons from a pair of siblings with BPAN
}

\author{
This article has been corrected since Advance Online Publication and a corrigendum is also printed in this issue
}

\author{
Yuri A Zarate ${ }^{\star,}$, Julie R Jones ${ }^{2}$, Melanie A Jones ${ }^{2}$, Francisca Millan ${ }^{3}$, Jane Juusola ${ }^{3}$, Annette Vertino-Bell ${ }^{3}$, \\ G Bradley Schaefer ${ }^{1}$ and Michael C Kruer ${ }^{4}$
}

Neurodegeneration with brain iron accumulation (NBIA) encompasses a heterogeneous group of inherited progressive neurological diseases. Beta-propeller protein-associated neurodegeneration (BPAN) has been estimated to account for $\sim 7 \%$ of all cases of NBIA and has distinctive clinical and brain imaging findings. Heterozygous variants in the WDR45 gene located in Xp11.23 are responsible for BPAN. A clear female predominance supports an X-linked dominant pattern of inheritance with proposed lethality for germline variants in hemizygous males. By whole-exome sequencing, we identified an in-frame deletion in the WDR45 gene (c.161_163delTGG) in the hemizygous state in a 20-year-old man with a history of profound neurocognitive impairment and seizures. His higher functioning 14-year-old sister, also with a history of intellectual disability, was found to carry the same variant in the heterozygous state. Their asymptomatic mother was mosaic for the alteration. From this pair of siblings with BPAN we conclude that: (1) inherited WDR45 variants are possible, albeit rare; (2) hemizygous germline variants in males can be viable, but likely result in a more severe phenotype; (3) for siblings with germline variants, males should be more significantly affected than females; and (4) because gonadal and germline mosaicism are possible and healthy female carriers can be found, parental testing for variants in WDR45 should be considered.

European Journal of Human Genetics (2016) 24, 1080-1083; doi:10.1038/ejhg.2015.242; published online 18 November 2015

\section{INTRODUCTION}

Neurodegeneration with brain iron accumulation (NBIA) encompasses a heterogeneous group of inherited, progressive neurological diseases characterized by cognitive impairment and prominent dystonia and/or parkinsonism. ${ }^{1-6}$ Beta-propeller protein-associated neurodegeneration (BPAN, NBIA5, OMIM: 300894) has been estimated to account for $\sim 7 \%$ of all cases of NBIA. ${ }^{7}$ Besides being the only type of NBIA with an X-linked dominant pattern of inheritance, BPAN has distinctive clinical and brain imaging findings. Its clinical course is described as biphasic with an initial presentation during childhood, as spastic quadriplegia or static encephalopathy with early developmental delay with or without seizures, followed by dementia in adulthood and the development of parkinsonism and dystonia. ${ }^{1,4,7-11}$

Heterozygous variants in the WD repeat-containing protein 45 gene (WDR45, OMIM: 300526) located in Xp11.23 lead to decreased autophagic activity and the BPAN phenotype. ${ }^{12}$ All well-described cases of BPAN in the literature have been sporadic with a clear female predominance supporting an X-linked dominant pattern of inheritance with proposed lethality in males with germline variants. We present only the fourth case of a hemizygous male and the first welldescribed brother-sister patient duo with a novel WDR45 in-frame deletion. The same variant was also detected in a mosaic state in their unaffected mother.

\section{MATERIALS AND METHODS}

Exome and Sanger sequencing

After extracting genomic DNA from whole blood from the affected patients and their parents, whole-exome sequencing was performed following standard techniques (Supplementary File 1). Variants were filtered based on inheritance patterns, gene lists of interest, phenotype and population frequencies, as appropriate. Resources including the Human Gene Mutation Database, 1000 Genomes database, NHLBI Exome Variant Server, OMIM, PubMed and ClinVar were used to evaluate genes and detect sequence changes of interest. Additional searches were performed using specific gene lists related to intellectual disability. The identified variant in WDR45 (according to NG_033004.1, NM_007075.3, see the Results section) was confirmed by conventional di-deoxy DNA sequence analysis using an ABI3730 (Life Technologies, Carlsbad, CA, USA) and standard protocols, and with allelespecific PCR with a new DNA preparation.

\section{Allele-specific PCR}

Allele-specific PCR was used to confirm the presence of the c.161_163delTGG variant allele in the mother. This confirmation method consisted of two reactions sharing the same reverse primer ( $5^{\prime}$-AGGCCTCCTCTCTGTGTGAC- $\left.3^{\prime}\right)$, but with a different forward primer that was complementary to either the wild-type or the variant allele. The forward primers were 5'-AGCATGGGCTTGGTGG-3' for the wild-type allele (TGG present) and $5^{\prime}$-CAGCATGGGCTTGGA $\overline{G-3^{\prime}}$ for the variant allele (TGG deleted). PCR products were imaged on a QIAxcel Advanced System (Qiagen Inc., Valencia, CA, USA).

\section{$\mathrm{X}$-chromosome inactivation studies}

The X-chromosome inactivation (XCI) pattern was determined by PCR analysis of a polymorphic CAG repeat in the first exon of the androgen receptor $(A R)$ gene. Methylation of sites close to this short tandem repeat have been demonstrated to correlate with X-chromosome inactivation. ${ }^{13}$ In this assay, amplification of the $A R$ gene both before and after digestion with the methylation-sensitive HpaII restriction enzyme was used to determine the methylation status of the maternal and paternal X chromosome. The inactive

${ }^{1}$ Section of Genetics and Metabolism, University of Arkansas for Medical Sciences, Arkansas Children's Hospital, Little Rock, AR, USA; ${ }^{6}$ Greenwood Genetic Center, Greenwood, SC, USA; ${ }^{3}$ GeneDx, Gaithersburg, MD, USA; ${ }^{4}$ Barrow Neurological Institute, Phoenix Children's Hospital, Phoenix, AZ, USA

${ }^{*}$ Correspondence: Dr YA Zarate, Section of Genetics and Metabolism, University of Arkansas for Medical Sciences, Arkansas Children's Hospital, 1 Children's Way, Slot 512-22 Little Rock, AR 72202, USA. Tel: +501 364 2971; Fax: +501 364 1564; E-mail: yazarate@uams.edu

Received 5 June 2015; revised 1 October 2015; accepted 13 October 2015; published online 18 November 2015 
$\mathrm{X}$ chromosome is highly methylated and prevents digestion at the HpaII site. Therefore, amplification of the $A R$ gene only occurs from the inactive $\mathrm{X}$ after HpaII digestion. XCI degree threshold patterns are classified as follows based on a ratio $\mathrm{X}_{1}: \mathrm{X}_{2}$ : random (below 80:20), moderately skewed (80:20-90:10) and highly skewed (above 90:10).

\section{Submission of variant details to public database}

The newly described WDR45 in-frame deletion data have been submitted to ClinVar with the accession number SCV000223914 (http://www.ncbi.nlm.nih. gov/clinvar/).

Clinical cases. Detailed clinical information about the patients is available in Supplementary File 1 . In brief, patient 1 is a 20 -year-old man with a history of global developmental delay and epilepsy. The patient was never able to walk independently, and although he is currently able to stand with support briefly, he is wheelchair dependent. There was no development of purposeful speech, and he is completely dependent for activities of daily living. During his most recent examination, his ears were large, measuring $6.8 \mathrm{~cm}(>2 \mathrm{SD})$, and he was noted to have multiple contractures with limited range of motion (Figure 1; Supplementary Video). At 20 years of age, his brain magnetic resonance imaging (MRI) revealed severe, diffuse brain atrophy and findings suggestive of iron deposition at the level of the globus pallidus and substantia nigra. His 14-year-old sister (patient 2) was evaluated for a long history of static encephalopathy. Developmental concerns appeared during the first year of life. At present, she only knows a few colors and can count up to 20 . She is able to dress and undress herself, use utensils, and speaks in short sentences. Throughout her life, no developmental regression has been documented. At 14 years of age, she was noted to have limited elbow extension, mild hirsutism and tapering of her fingers (Figure 1; Supplementary Video). A brain MRI at that time revealed iron deposition in the basal ganglia.

The mother of patients 1 and 2 (patient 3 ) is an otherwise healthy 46-yearold Mexican woman. The family history was unremarkable for developmental delay, seizures or other possible neurodegenerative conditions. She denies any ongoing health issues and has no previous cognitive or developmental concerns. She has not had any seizures and so far has no neurological sign or symptom. There has not been a medical reason to justify ordering brain images on her.

XCI and whole-exome sequencing results. The XCI studies revealed a highly skewed pattern of inactivation of 92:8 in patient 2. On the basis of the $\mathrm{X}$-inactivation data, and assuming no recombination occurred between the WDR45 gene and the AR locus, the patient was found to be skewed towards the maternal WDR45 allele, with the abnormal allele being preferentially active. The mother's XCI was found to be random with a ratio of 63:37.

A novel in-frame deletion in the WDR45 gene (c.161_163delTGG, p.(Val54del)) was detected in the hemizygous, heterozygous and mosaic states in the brother, sister and mother, respectively. The variant was not found in the father of patients 1 and 2. Sanger sequencing confirmed the presence of the deletion in the proband and sister but not in the mother, most likely because it was present at a level below the limit of detection by sequencing. Allele-specific PCR confirmed the presence of the mutant allele in the mother in a somatic mosaic state (Figure 2). The c.161_163delTGG variant identified in this family results in a deletion of a conserved valine amino acid at codon 54 . The variant was not observed in 6500 individuals of European and African American ancestry in the NHLBI Exome Sequencing Project (http://evs.gs.washington. edu/EVS/) and was absent in the Exome Aggregation Consortium database (http://exac.broadinstitute.org/, accessed August 2015), a large compilation of
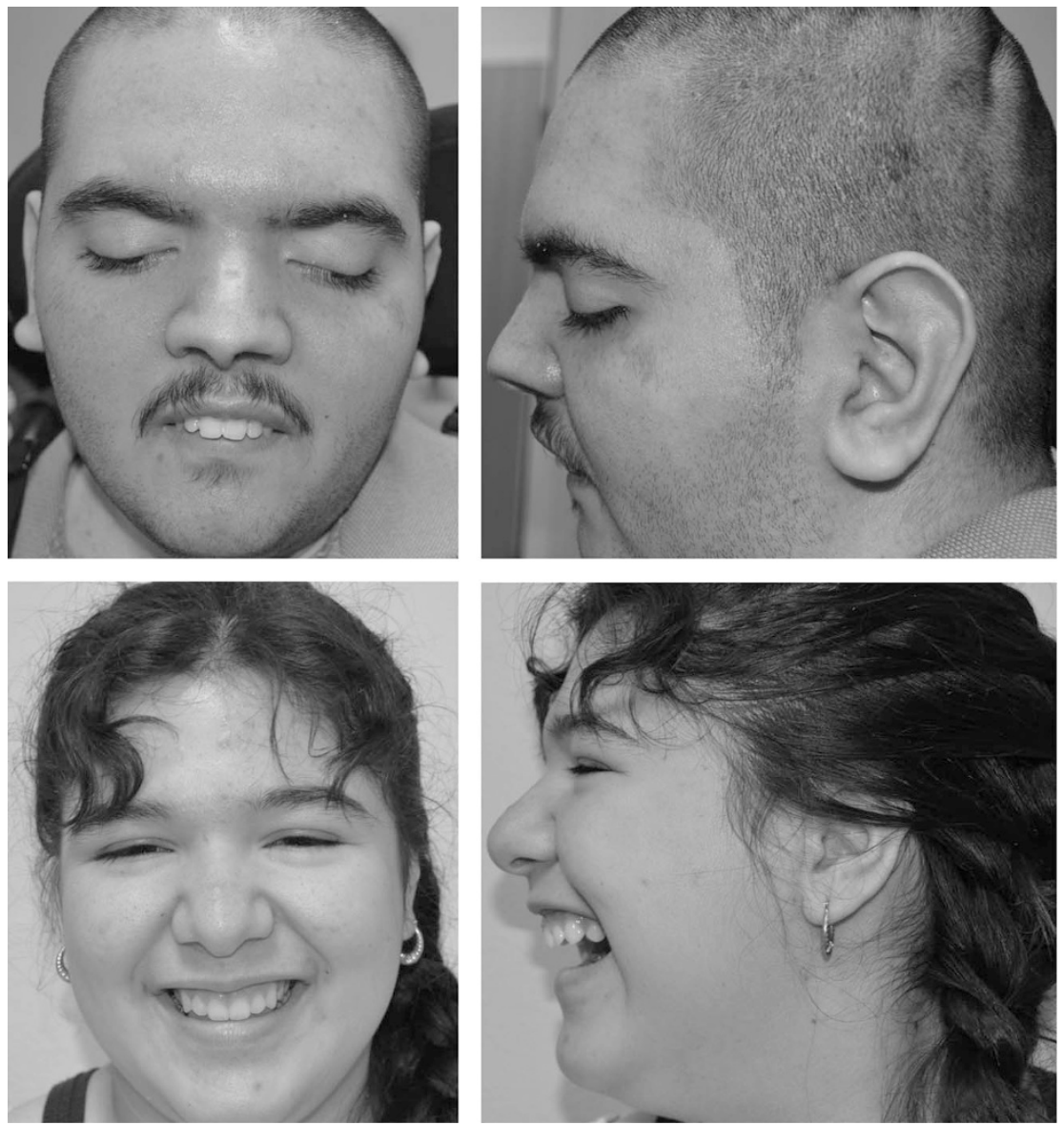

Figure 1 Top: patient 1 at 20 years of age. Note the high nasal bridge, downslanted palpebral fissures and large ears with prominent inferior crus of the antihelix. Bottom: patient 2 at 13 years of age. Note high nasal bridge and low hanging columella. 

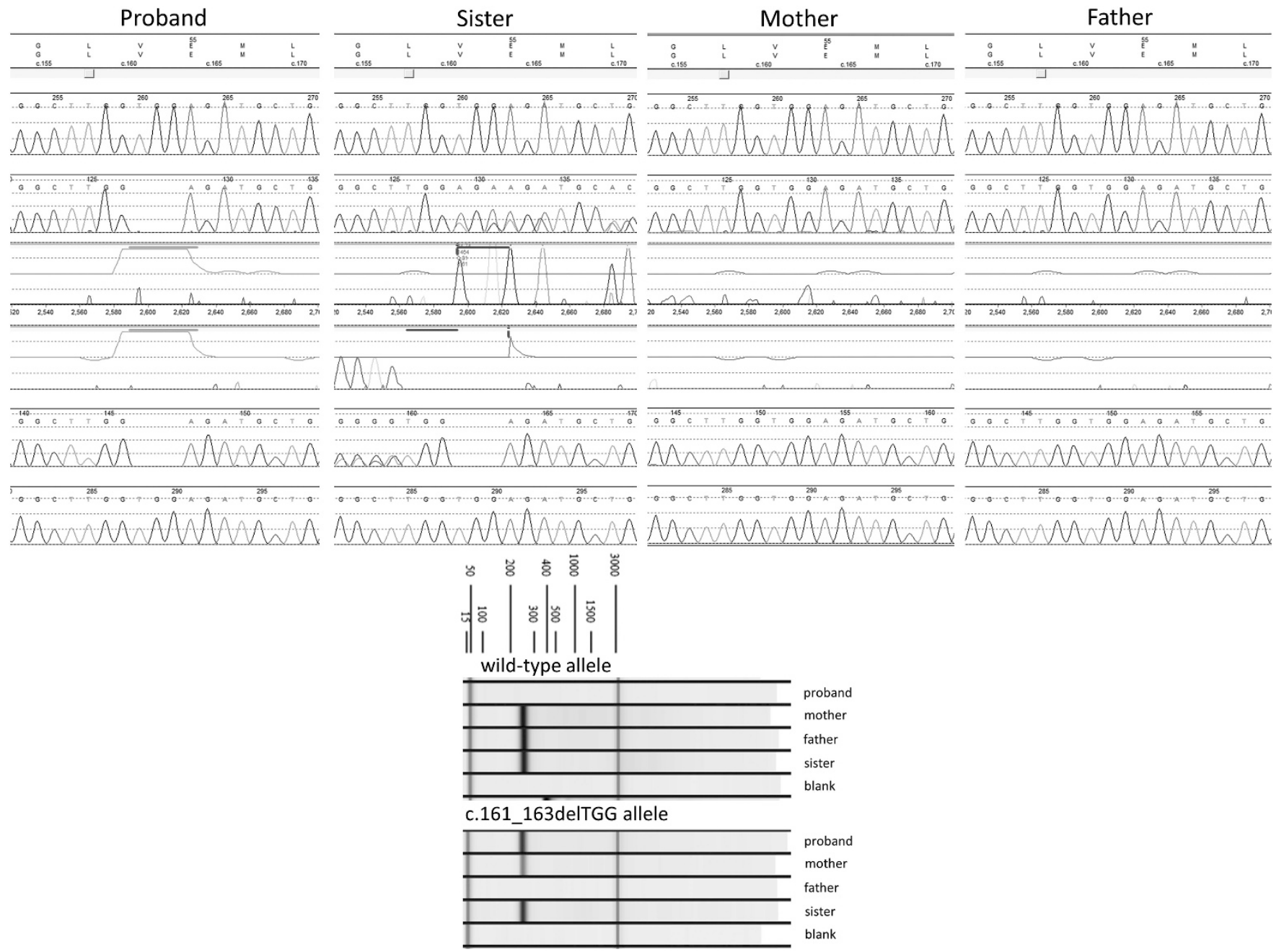

Figure 2 Top: Sanger sequencing confirmed the WDR45 p.(Val54del; c.161_163delTGG) variant in the hemizygous and heterozygous states in the proband and his sister. The deletion was not detected in either parent by this method. Bottom: Allele-specific PCR demonstrates the presence of the variant allele in the mother. The relative intensity of the band is consistent with its presence at a lower level than in her children, confirming somatic mosaicism.

sequencing data from over 60000 unrelated individuals that includes several thousand Latino samples.

\section{DISCUSSION}

Only one other patient with BPAN has been previously reported to have an in-frame deletion in the WDR45 gene: c.752_754del, p.(Ser251del). ${ }^{14}$ To the best of our knowledge, no benign, in-frame deletions have been reported in the WDR45 gene in any of the publicly available databases. This report of familial cases of BPAN offers other valuable lessons when compared with all the cases described in the literature. All well-described cases of BPAN in the literature to date had been sporadic, suggesting that the majority are a result of de novo variants early in development. From this case, we can conclude that although de novo variants early in development are the main mechanism, inherited variants are possible, albeit rare.

Hemizygous germline variants in males have been presumed to be nonviable. ${ }^{1,3,7,10,12,15}$ Here we have demonstrated that although hemizygous germline variants in males are not universally lethal as it was originally presumed, they can result in a more severe phenotype. The direct clinical comparison of the two siblings here described revealed a more significant neurodevelopmental compromise at all ages in the male proband compared with his sister. Therefore, we can expect that for siblings with germline variants, males should be more significantly affected than females, although the degree of autophagy functional deficits that allow survival in the former and the XCI skewing in the latter, affect the severity of the phenotype. The meaning of XCI skewing favoring the mutated allele, as seen in the proband's sister, or biallelic expression of both wild-type and mutant alleles as has been previously reported, remains unclear. ${ }^{12,16}$

In 2013, Hayflick et al. ${ }^{10}$ recommended that because gonadal or germline mosaicism was a theoretical possibility, parental testing for variants in WDR45 should be considered. In agreement with this early suggestion and despite its rarity, the demonstration that maternal gonadal and germline mosaicism is possible supports this argument. Although no brain imaging has been performed, the lack of signs or symptoms at the age of assessment in the proband's mother indicates that healthy female carriers also exist. Therefore, cases should not be assumed to be de novo when clinical signs are not present in the mother.

\section{CONFLICT OF INTEREST}

The authors declare no conflict of interest. 


\section{ACKNOWLEDGEMENTS}

We would like to thank Megan Cho, CGC, for her logistic assistance and for facilitating the communication between clinicians and the diagnostic laboratory, and Dr Tom Collins II, MD, for his critical review of the manuscript and suggestions.

1 Doorn JM, Kruer MC: Newly characterized forms of neurodegeneration with brain iron accumulation. Curr Neurol Neurosci Rep 2013; 13: 413.

2 Van Goethem G, Livingston JH, Warren D, Oojageer AJ, Rice GI, Crow YJ: Basal ganglia calcification in a patient with beta-propeller protein-associated neurodegeneration. Pediatr Neurol 2014; 51: 843-845.

3 Haack TB, Hogarth P, Kruer MC et al: Exome sequencing reveals de novo WDR45 mutations causing a phenotypically distinct, X-linked dominant form of NBIA. Am J Hum Genet 2012; 91: 1144-1149.

4 Kruer MC, Boddaert N: Neurodegeneration with brain iron accumulation: a diagnostic algorithm. Semin Pediatr Neurol 2012; 19: 67-74.

5 Kruer MC, Boddaert N, Schneider SA et al: Neuroimaging features of neurodegeneration with brain iron accumulation. Am J Neuroradiol 2012; 33: 407-414.

6 Levi S, Finazzi D: Neurodegeneration with brain iron accumulation: update on pathogenic mechanisms. Front Pharmacol 2014; 5: 99.

7 Hogarth P: Neurodegeneration with brain iron accumulation: diagnosis and management. J Mov Disord 2015; 8: 1-13.
8 Gregory A, Hayflick SJ: Genetics of neurodegeneration with brain iron accumulation. Curr Neurol Neurosci Rep 2011; 11: 254-261.

9 Gregory A, Polster BJ, Hayflick SJ: Clinical and genetic delineation of neurodegeneration with brain iron accumulation. J Med Genet 2009; 46: 73-80.

10 Hayflick SJ, Kruer MC, Gregory A et al: beta-Propeller protein-associated neurodegeneration: a new X-linked dominant disorder with brain iron accumulation. Brain 2013; 136: 1708-1717.

11 Schneider SA, Dusek P, Hardy J, Westenberger A, Jankovic J, Bhatia KP: Genetics and Pathophysiology of Neurodegeneration with Brain Iron Accumulation (NBIA). Curr Neuropharmacol 2013; 11: 59-79.

12 Saitsu H, Nishimura T, Muramatsu $\mathrm{K}$ et al: De novo mutations in the autophagy gene WDR45 cause static encephalopathy of childhood with neurodegeneration in adulthood. Nat Genet 2013; 45: 445-449.

13 Allen RC, Zoghbi HY, Moseley AB, Rosenblatt HM, Belmont JW: Methylation of Hpall and Hhal sites near the polymorphic CAG repeat in the human androgen-receptor gene correlates with X chromosome inactivation. Am J Hum Genet 1992; 51 . 1229-1239.

14 Verhoeven WM, Egger JI, Koolen DA et al: Beta-propeller protein-associated neurodegeneration (BPAN), a rare form of NBIA: novel mutations and neuropsychiatric phenotype in three adult patients. Parkinsonism Relat Disord 2014; 20: 332-336.

15 Haack TB, Hogarth P, Gregory A, Prokisch H, Hayflick SJ: BPAN: the only X-linked dominant NBIA disorder. Int Rev Neurobiol 2013; 110: 85-90.

16 Nishioka K, Oyama G, Yoshino $\mathrm{H}$ et al: High frequency of beta-propeller proteinassociated neurodegeneration (BPAN) among patients with intellectual disability and young-onset parkinsonism. Neurobiol Aging 2015; 36: 2004.e9-2004.e15.

Supplementary Information accompanies this paper on European Journal of Human Genetics website (http://www.nature.com/ejhg) 